\title{
Influência do uso simultâneo de ácido ascórbico e azodicarbonamida na qualidade do pão francês
}

The influence of simultaneous use of ascorbic acid and azodicarbonamide in the quality of french bread

\author{
Alessandra Santos LOPES ${ }^{1}$, Rita de Cássia Salvucci Celeste ORMENESE ${ }^{1}$, \\ Flávio Martins MONTENEGRO ${ }^{*}$, Patrocínio Gonçalves FERREIRA JÚNIOR ${ }^{1}$
}

\begin{abstract}
Resumo
Este trabalho teve como objetivo avaliar o uso simultâneo de ácido ascórbico e azodicarbonamida em produto de panificação e através da metodologia de superfície de resposta. As respostas do planejamento experimental $\left(2^{2}\right)$ foram: o volume específico e a pontuação total das características externas e internas do pão francês. A ação do ácido ascórbico no aumento do volume específico do pão francês teve efeitos significativos ( $\mathrm{p} \leq 0,05$ ou valores próximos) linear e quadrático. Para a azodicarbonamida verificou-se somente efeito quadrático ( $\mathrm{p} \leq 0,05$ ou valores próximos), e não houve qualquer efeito de interação entre os dois agentes oxidantes estudados. A aplicação de ácido ascórbico e azodicarbonamida em concentrações acima de $75 \mathrm{mg} . \mathrm{kg}^{-1}$ de farinha de trigo e $30 \mathrm{mg} \cdot \mathrm{kg}^{-1}$, dentro dos níveis estudados, proporcionou a produção de pães franceses de maior volume específico.

Palavras-chave: pão; ácido ascórbico; azodicarbonamida; planejamento experimental.
\end{abstract}

\begin{abstract}
The aim of this work was to evaluate the simultaneous use of ascorbic acid and azodicarbonamide in a bakery product by using the response of the surface methodology. The experimental design $\left(2^{2}\right)$ responses were the specific volume and total count of the external and internal characteristics of French bread. The action of ascorbic acid on the increase of the specific volume of French bread had significant linear and quadratic effects ( $\mathrm{p} \leq 0.05$ or near values). For azodicarbonamide, the quadratic effect was observed, and there was no interaction effect between the two studied oxidants agents. The application of ascorbic acid and azodicarbonamide using concentrations above 75 mg.kg ${ }^{-1}$ of wheat flour and 30mg. $\mathrm{kg}^{-1}$, considering the studied levels, provided French bread production with a higher specific volume.

Keywords: bread; ascorbic acid; azodicarbonamide; experimental design.
\end{abstract}

\section{Introdução}

Condicionadores de massa para panificação têm sido utilizados como aditivos em produtos de padaria por quase cinqüenta anos, visando melhorar suas características durante o amassamento, a qualidade sensorial e a vida de prateleira ${ }^{11,15}$. Os condicionadores de massa podem ser agrupados dentro de quatro categorias: emulsificantes (estearoil-2-lactilato de sódio ou de cálcio e polisorbato), oxidantes (bromato de potássio), redutores (L-cisteína) e redutores de tempo de mistura (proteases) ${ }^{12,15,18}$.

De acordo com DANNO e HOSENEY ${ }^{6}$, o tipo e a quantidade do condicionador de massa influenciam as características reológicas da massa viscoelástica obtida da mistura de farinha de trigo e água. Segundo STAUFFER ${ }^{16}$, os condicionadores que reforçam a rede de glúten, em sua grande maioria, são compostos por agentes oxidantes, como o ácido ascórbico, bromato de potássio e azodicarbonamida.

FITCHETT e FRAZIER ${ }^{11}$ mencionaram que a azodicarbonamida (ADA) proporcionou a diminuição do tempo de desenvolvimento da massa e a redução da tolerância à sobremistura. Também verificaram que massas contendo ácido L-ascórbico (AA) foram mais tolerantes para tempos maiores ou menores de mistura, e que o uso de L-cisteína diminuiu o requerimento de energia para atingir o desenvolvimento ótimo da massa.

Recebido para publicação em 19/6/2006

Aceito para publicação em 23/4/2007 (001766)

${ }^{1}$ Instituto de Tecnologia de Alimentos - ITAL/CEREAL, CHOCOTEC,

Avenida Brasil 2880, CEP 13070-178, Campinas - SP, Brasil,

E-mail: flavio@ital.sp.gov.br

*A quem a correspondência deve ser enviada

O ácido ascórbico tem sido amplamente usado como oxidante na indústria de panificação. A quantidade empregada para um bom desempenho no processamento da massa varia de 10-200 mg. $\mathrm{kg}^{-1}$ (em relação ao peso de farinha), dependendo do efeito requerido na qualidade final dos produtos de panificação ${ }^{9,11,17}$.

FISHER ${ }^{10}$ apresentou valores relativos aos níveis máximos de uso e à velocidade de ação dos agentes oxidantes disponíveis para a panificação. Foi verificado que a azodicarbonamida e o ácido ascórbico possuem velocidade de ação rápida, e a aplicação desses agentes oxidantes ocasiona a redução da extensibilidade e o aumento da retenção dos gases, resultando um maior volume e melhoria na granulosidade e na textura do pão.

EL-HADY et al. ${ }^{9}$ verificaram que o uso combinado de ácido L-ascórbico e bromato de potássio teve efeito melhorador mais pronunciado que a adição de ácido L-ascórbico isoladamente. WOOD $^{19}$ cita o uso de outros oxidantes empregados como condicionadores de massa para panificação, tais como a azodicarbonamida e o iodato de potássio.

A qualidade do pão é normalmente determinada pela avaliação das características externas, onde se inclui o volume específico, a cor da crosta, a quebra e a simetria, pelas características internas de característica da crosta, cor do miolo, célula do miolo e textura do miolo, além de aroma e sabor ${ }^{8,13}$.

O objetivo deste trabalho foi avaliar o efeito do uso simultâneo de ácido L-ascórbico e azodicarbonamida nos parâmetros de qualidade de pão francês (volume específico e características 
externas e internas) aplicando a metodologia de superfície de resposta.

\section{Material e métodos}

\subsection{Análises da farinha de trigo}

A farinha de trigo utilizada neste estudo foi avaliada de acordo com as análises de teor de umidade, teor de glúten úmido e seco, índice de glúten, atividade enzimática através do Falling Number, características farinográficas e extensográficas, citadas abaixo:

Teor de umidade: determinado segundo o método 44-15A da $\mathrm{AACC}^{1}$.

Teor de glúten úmido e seco e índice de glúten: determinados de acordo com o método 38-12 da AACC $^{1}$.

Atividade enzimática: determinada em aparelho Falling Number através do método 56-81B da $\mathrm{AACC}^{1}$.

Absorção de água e propriedades de mistura (tempo de chegada, tempo de desenvolvimento, tempo de saída, estabilidade e índice de tolerância à mistura) foram avaliados em farinógrafo Brabender, segundo método 54-21 da $\mathrm{AACC}^{1}$.

As propriedades de extensão da farinha de trigo (extensibilidade, resistência à extensão ou elasticidade, resistência máxima, energia, número proporcional e número de oxidação) foram determinadas em extensógrafo Brabender, segundo método 54-10 da $\mathrm{AACC}^{1}$.

\subsection{Matéria-prima, aditivos e formulação da massa de pão francês}

A formulação básica empregada na produção das massas de pão tipo francês é apresentada na Tabela 1. Nesta formulação foram aplicados os agentes oxidantes: ácido L-ascórbico cristalino e azodicarbonamida. De acordo com o planejamento experimental (Tabela 2) foram utilizados teores de ácido Lascórbico de 14,65 a 85,35 mg. $\mathrm{kg}^{-1}$ e de 6 a 34 mg. $\mathrm{kg}^{-1}$ de azodicarbonamida, em relação ao peso de farinha de trigo.

Tabela 1. Formulação básica da massa de pão francês.

\begin{tabular}{lc}
\hline \multicolumn{1}{c}{ Ingredientes } & Quantidade (\%) \\
\hline Farinha de trigo & 100,0 \\
Água & 64,9 \\
Fermento biológico seco instantâneo & 1,0 \\
Sal refinado & 2,0 \\
Açúcar refinado & 0,5 \\
Polisorbato 80 & 0,3 \\
DATEM & 0,03 \\
CSL & 0,07
\end{tabular}

*Em relação ao total de farinha de trigo.

A avaliação do efeito da adição simultânea de ácido ascórbico e azodicarbonamida foi realizada segundo um planejamento composto central $\left(2^{2}\right)$, constituído por quatro ensaios lineares nos níveis -1 e +1 , quatro ensaios axiais $(\alpha=1,414)$ e três ensaios no ponto central, como pode ser observado na Tabela 3.
As variáveis independentes foram: teor de ácido L-ascórbico (mg. $\mathrm{kg}^{-1}$ ) e de azodicarbonamida (mg. $\mathrm{kg}^{-1}$ ), sendo seus níveis apresentados na Tabela 2 . As respostas do planejamento foram: o volume específico multiplicado pelo fator 3,33 e as características externas e internas do pão, de acordo com El-Dash ${ }^{8}$. Utilizando-se o programa Statistica ${ }^{\circledR}$ versão $5.0^{14}$, os resultados foram avaliados através da verificação dos efeitos significativos ( $\mathrm{p} \leq$ 0,05 ou valores próximos), análise de variância (ANOVA) e Metodologia de Superfície de Resposta.

Tabela 2. Variáveis independentes e seus diferentes níveis.

\begin{tabular}{lcccccc}
\hline \multicolumn{1}{c}{ Fator } & Símbolo & \multicolumn{5}{c}{ Níveis } \\
\cline { 3 - 7 } & & $-\alpha$ & -1 & 0 & +1 & $+\alpha$ \\
\hline $\begin{array}{l}\text { Ácido L-ascórbico } \\
\text { (mg.kg-1) }\end{array}$ & $\mathrm{X} 1$ & 14,65 & 25,00 & 50,00 & 75,00 & 85,35 \\
$\begin{array}{l}\text { Azodicarbonamida } \\
\left(\mathrm{mg}_{\mathrm{kg}}^{-1} \text { ) }\right.\end{array}$ & $\mathrm{X} 2$ & 6 & 10 & 20 & 30 & 34 \\
\hline
\end{tabular}

Tabela 3. Planejamento experimental completo $\left(2^{2}\right)$.

\begin{tabular}{|c|c|c|}
\hline Ensaio & $\mathrm{X} 1$ & $\mathrm{X} 2$ \\
\hline 1 & -1 & -1 \\
\hline 2 & 1 & -1 \\
\hline 3 & -1 & 1 \\
\hline 4 & 1 & 1 \\
\hline 5 & $-1,414$ & 0 \\
\hline 6 & 1,414 & 0 \\
\hline 7 & 0 & $-1,414$ \\
\hline 8 & 0 & 1,414 \\
\hline $9(\mathrm{C})$ & 0 & 0 \\
\hline $10(\mathrm{C})$ & 0 & 0 \\
\hline $11(\mathrm{C})$ & 0 & 0 \\
\hline
\end{tabular}

\subsection{Processo de fabricação do pão francês}

O pão francês foi obtido através do método massa direta e consistiu da mistura dos ingredientes usados na formulação em masseira com haste tipo gancho, incluindo o ácido L-ascórbico e a azodicarbonamida. Essa etapa foi realizada em duas fases: velocidade lenta por 9 minutos e velocidade rápida por 7 minutos, até o completo desenvolvimento do glúten. Foi utilizada água na temperatura de 10 a $12{ }^{\circ} \mathrm{C}$ para obtenção de massa com temperatura final de 26 a $28{ }^{\circ} \mathrm{C}$. Após a mistura, a massa foi dividida em porções de $60 \mathrm{~g}$, boleadas manualmente, moldadas no formato de pão francês, e dispostas em formas de tela metálica. O tempo decorrido após a mistura da massa e o início da moldagem foi de 20 minutos, sendo esse o tempo de espera ou descanso da massa, fixado para todos os ensaios. As massas, já moldadas, foram levadas à câmara de fermentação com controle de temperatura (30 a $32{ }^{\circ} \mathrm{C}$ ) e umidade relativa ( 80 a $90 \%$ ), pelo período de 1:45 h, onde foi observado um crescimento no volume de aproximadamente duas vezes. Finalmente, após a fermentação realizou-se o corte da superfície da massa e o assamento em forno elétrico por 16 minutos a $200{ }^{\circ} \mathrm{C}$. 


\section{Resultados e discussão}

\subsection{Análises da farinha de trigo}

A farinha de trigo utilizada nos experimentos apresentou teor de umidade médio de 13,36\% (Tabela 4), estando de acordo com a legislação $0^{5}$ que determina o limite máximo de $15 \%$. Segundo os valores médios de índice de glúten $(97,38)$ e Falling Number (304 s), a farinha de trigo pode ser classificada como adequada para a produção de pães de boa qualidade tecnológica ${ }^{2}$.

De acordo com ATWELL2 ${ }^{2}$, geralmente, farinhas com características adequadas à produção de pães apresentam alta absorção de água, tempo de desenvolvimento elevado e uma certa tolerância ao excesso de mistura.

Considerando os parâmetros farinográficos (Tabela 5) e extensográficos (Tabela 6), a farinha de trigo pode ser classificada como forte, portanto, pode ser aplicada para uso em panificação.

\subsection{Efeito dos agentes oxidantes no volume específico e na pontuação total das características externas e internas do pão francês}

As médias do volume específico (mL.g $\left.{ }^{-1}\right)$ e da pontuação total das características externas e internas do pão francês são mostradas na Tabela 7 .

\section{Volume específico}

Os resultados da análise estatística aplicada aos dados de volume específico, de acordo com o erro puro e a soma quadrática residual, são mostrados nas Tabelas 8 e 9 . Os efeitos dos fatores lineares, quadráticos e da interação, em negrito, são significativos em nível de $95 \%$ de confiança $(p \leq 0,05)$ ou valores próximos.

Como podem ser observados nas Tabelas 8 e 9, os efeitos significativos a $95 \%$ de confiança, considerando o erro puro e a soma quadrática residual, foram $\mathrm{AA}(\mathrm{L})$ e $\mathrm{AZO}(\mathrm{Q})$. No entanto, o efeito $\mathrm{AA}(\mathrm{Q})$ apresentou significância estatística dentro de

Tabela 4. Umidade, teor de glúten úmido e seco, índice de glúten e falling number.

\begin{tabular}{cccccc}
\hline Amostra & Umidade * (\%) $^{*}$ & Glúten úmido* $(\%)$ & Glúten seco* $(\%)$ & Índice de glúten* & Falling number* ${ }^{*}$ (s) \\
\hline Farinha padrão & $13,36 \pm 0,04$ & $28,60 \pm 1,27$ & $9,90 \pm 0,57$ & $97,38 \pm 0,13$ & $304 \pm 5,7$ \\
\hline *
\end{tabular}

*Média \pm desvio padrão de três determinaçōes.

Tabela 5. Parâmetros farinográficos da farinha de trigo ( $14 \%$ de umidade).

\begin{tabular}{|c|c|c|c|c|c|c|}
\hline Amostra & ABS* (\%) & $\mathrm{TC}^{*}(\mathrm{~min})$ & TDM* (minutos) & TS* (minutos) & $\mathrm{EST}^{*}$ (minutos) & ITM* (UB) \\
\hline Farinha padrão & $63,8 \pm 0,42$ & $1,25 \pm 0,35$ & $5,5 \pm 0,0$ & $11,0 \pm 1,41$ & $12,25 \pm 1,06$ & $35,0 \pm 7,07$ \\
\hline
\end{tabular}

*Média \pm desvio padrão de três determinações; $\mathrm{ABS}=$ absorção de água; $\mathrm{TC}=$ tempo de chegada; TDM = tempo de desenvolvimento da massa; TS = tempo de saída; EST = estabilidade; ITM = índice de tolerância à mistura (em unidades Brabender - U.B.).

Tabela 6. Parâmetros extensográficos da farinha de trigo ( $14 \%$ de umidade) relativos ao tempo de descanso de 45,90 e 135 minutos.

\begin{tabular}{ccccccc}
\hline Amostra & \multicolumn{1}{c}{$\mathrm{E}^{*}(\mathrm{~mm})$} & \multicolumn{1}{c}{$\mathrm{R}^{*}$ (U.B.) } & \multicolumn{1}{c}{ Rm* (U.B.) } & \multicolumn{1}{c}{$\mathrm{A}^{*}\left(\mathrm{~cm}^{2}\right)$} & $\mathrm{D}^{*}$ & Nox $^{*}$ \\
\hline 45 minutos & $147,5 \pm 1,41$ & $395,0 \pm 7,07$ & $530,0 \pm 7,07$ & $107,03 \pm 0,66$ & $2,68 \pm 0,02$ & $39,91 \pm 0,48$ \\
90 minutos & $123,25 \pm 4,60$ & $667,50 \pm 17,7$ & $817,50 \pm 38,9$ & $131,61 \pm 10,7$ & $5,42 \pm 0,34$ & $24,39 \pm 3,54$ \\
135 minutos & $105,5 \pm 7,07$ & $782,5 \pm 60,1$ & $835,0 \pm 63,6$ & $111,8 \pm 0,6$ & $7,46 \pm 1,06$ & $15,17 \pm 2,3$ \\
\hline
\end{tabular}

*Média \pm desvio padrão de três determinações; $\mathrm{E}=$ extensibilidade; $\mathrm{R}=$ resistência à extensão (em unidades Brabender); $\mathrm{Rm}=$ resistência máxima; $\mathrm{A}=$ energia; $\mathrm{D}=$ número proporcional $(\mathrm{R} / \mathrm{E}) ;$ e Nox $=$ número de oxidação $(\mathrm{AE} / \mathrm{R})$.

Tabela 7. Volume específico e pontuação total das características externas e internas do pão francês de acordo com o planejamento experimental.

\begin{tabular}{|c|c|c|c|c|c|c|}
\hline \multirow[t]{2}{*}{ Ensaio } & \multicolumn{2}{|c|}{ Codificado } & \multicolumn{2}{|c|}{ Não codificado* } & \multirow[t]{2}{*}{ Volume específico x 3,33 $\left(\mathrm{ml} \cdot \mathrm{g}^{-1}\right)$} & \multirow[t]{2}{*}{ Pontuação total } \\
\hline & $\mathrm{X} 1$ & $\mathrm{X} 2$ & $\mathrm{AA}\left(\mathrm{mg} \cdot \mathrm{kg}^{-1}\right)^{1}$ & $\mathrm{AZO}\left(\mathrm{mg} \cdot \mathrm{kg}^{-1}\right)^{2}$ & & \\
\hline 1 & -1 & -1 & 25,00 & 10 & 17,537 & 82,09 \\
\hline 2 & -1 & 1 & 25,00 & 30 & 17,843 & 82,34 \\
\hline 3 & 1 & -1 & 75,00 & 10 & 19,281 & 83,28 \\
\hline 4 & 1 & 1 & 75,00 & 30 & 20,000 & 84,50 \\
\hline 5 & $-1,414$ & 0 & 14,65 & 20 & 16,648 & 78,65 \\
\hline 6 & 1,414 & 0 & 85,35 & 20 & 19,251 & 82,25 \\
\hline 7 & 0 & $-1,414$ & 50,00 & 6 & 18,862 & 82,36 \\
\hline 8 & 0 & 1,414 & 50,00 & 34 & 18,962 & 82,46 \\
\hline 9 & 0 & 0 & 50,00 & 20 & 17,776 & 81,78 \\
\hline 10 & 0 & 0 & 50,00 & 20 & 17,438 & 83,44 \\
\hline 11 & 0 & 0 & 50,00 & 20 & 17,461 & 82,46 \\
\hline
\end{tabular}

${ }^{1} \mathrm{AA}=$ ácido ascórbico; ${ }^{2} \mathrm{AZO}=$ azodicarbonamida; e *em relação ao peso de farinha de trigo. 
Tabela 8. Efeito estimado, erro puro, coeficiente t e significância estatística de cada fator para o volume específico do pão francês.

\begin{tabular}{|c|c|c|c|c|}
\hline Fatores & Efeito estimado & Erro puro & $\mathrm{t}(2)$ & Significância estatística (p) \\
\hline \multicolumn{5}{|l|}{ Efeitos principais } \\
\hline $\mathrm{AA}(\mathrm{L})$ & 1,8955 & 0,1335 & 14,1945 & 0,0049 \\
\hline AA (Q) & 0,5084 & 0,1589 & 3,1987 & 0,0854 \\
\hline $\mathrm{AZO}(\mathrm{L})$ & 0,2916 & 0,1335 & 2,1836 & 0,1607 \\
\hline $\mathrm{AZO}(\mathrm{Q})$ & 1,4709 & 0,1589 & 9,2542 & 0,0115 \\
\hline \multicolumn{5}{|l|}{ Efeito de interação } \\
\hline $\mathrm{AA}(\mathrm{L}) \times \mathrm{AZO}(\mathrm{L})$ & 0,2065 & 0,1888 & 1,0934 & 0,3883 \\
\hline
\end{tabular}

AA: ácido ascórbico; AZO: azodicarbonamida; (L): linear; e (Q): quadrático.

Tabela 9. Efeito estimado, soma quadrática residual, coeficiente t e significância estatística de cada fator para o volume específico do pão francês.

\begin{tabular}{cccrc}
\hline Fatores & Efeito estimado & Soma quadrática residual & $\mathrm{t}(5)$ & Significância estatística (p) \\
\cline { 1 - 4 } Efeitos principais & & & 11,2294 & 0,0001 \\
AA (L) & 1,8955 & 0,1688 & 2,5305 & 0,0525 \\
AA (Q) & 0,5084 & 0,2009 & 1,7275 & 0,1446 \\
AZO (L) & 0,2916 & 0,1688 & 0,0007 \\
AZO (Q) & 1,4709 & 0,2009 & 7,3211 & 0,4266 \\
\hline Efeito de interação & & & & 0,8650 \\
\hline AA (L) x AZO (L) & 0,2065 & 0,2387 & & \\
\hline
\end{tabular}

AA: ácido ascórbico; AZO: azodicarbonamida; (L): linear; e (Q): quadrático.

um limite de confiança próximo a 95\%, e por isso foi avaliado como significativo.

O efeito estimado indica o quanto cada fator influi na resposta estudada, pois quanto maior o seu valor em módulo, maior é a sua influência. Um efeito positivo indica que o aumento da variável provoca um aumento na resposta, já o efeito negativo indica que para um aumento da variável ocorre uma diminuição na resposta. Diante disso, pode-se verificar que o efeito AA(L) apresentou maior influência no volume específico do pão francês.

Após a eliminação dos parâmetros com efeitos não significativos $\mathrm{AZO}(\mathrm{L})$ e a interação $\mathrm{AA}(\mathrm{L}) \times \mathrm{AZO}(\mathrm{L})$, foi realizada a análise de variância (ANOVA) e verificada a significância da regressão e da falta de ajuste em nível de 95\% de confiança ( $\mathrm{p} \leq 0,05)$, utilizando o teste F, conforme a Tabela 10 .

Segundo BARROS NETO, SCARMINIO e BRUNS ${ }^{3}$, nem sempre uma regressão dada como significativa pelo teste $\mathrm{F}$ é útil para realizar previsões, pois pode acontecer que a faixa de variação coberta pelos fatores estudados seja pequena demais. De acordo com BOX e WETZ citados por BARROS NETO et al. ${ }^{3}$, uma regra prática que pode ser empregada é a de considerar a regressão como útil para fins preditivos caso o valor $\mathrm{MQ}_{\text {regressão }} / \mathrm{MQ}_{\text {resíduo }}$ seja, pelo menos, quatro a cinco vezes maior que o $\mathrm{F}_{\text {tabelado }}$, e o $\mathrm{F}$ da falta de ajuste em relação ao erro puro deve apresentar o menor valor possível e sempre menor que o $\mathrm{F}$ da falta de ajuste tabelado, pois um valor alto e maior que o $\mathrm{F}_{\text {tabelado }}$ para a falta de ajuste indica que os dados não estão bem ajustados ao modelo obtido.

Como se pode observar na Tabela 10, o modelo apresentou regressão significativa em nível de $95 \%$ de confiança ( $\mathrm{F}_{\text {calculado }}$ superior ao $\mathrm{F}_{\text {tabelado }}$ ) com $\mathrm{R}^{2}$ igual a 0,9537 , evidenciando que o modelo explicou 95,37\% da variação dos dados experimentais. $\mathrm{O}$ valor de $\mathrm{F}_{\text {calculado }}$ da regressão foi 11,0 vezes maior que o $\mathrm{F}_{\text {tabelado, }}$, indicando que o modelo é preditivo. A falta de ajuste não foi significativa ( $\mathrm{F}_{\text {calculado }}$ menor que $\mathrm{o} \mathrm{F}_{\text {tabelado }}$ ), e isso mostra que os dados experimentais ajustaram-se ao modelo obtido.

O modelo proposto para representar o volume específico do pão francês em função da adição de ácido ascórbico e azodicarbonamida é o seguinte:

$\mathrm{VE}=17,558+0,948 \times \mathrm{AA}+0,254 \times \mathrm{AA}^{2}+0,736 \times \mathrm{AZO}^{2}$

em que, $\mathrm{AA}=$ ácido ascórbico $\left(\mathrm{mg}^{\mathrm{kg}}{ }^{-1}\right) ; \mathrm{AZO}=$ azodicarbonamida (mg.kg- $\left.{ }^{-1}\right) ; \mathrm{VE}=$ volume específico $\left(\mathrm{mL} \cdot \mathrm{g}^{-1}\right)$.

A Figura 1 mostra a superfície de resposta quadrática (a) e as curvas de nível (b) geradas através do modelo proposto.

A superfície de resposta quadrática e as curvas de nível confirmam a análise de efeitos e permitem visualizar a variação

Tabela 10. Análise de variância (ANOVA) do modelo ajustado para o volume específico do pão francês.

\begin{tabular}{|c|c|c|c|c|c|c|}
\hline Fonte de variação & SQ & GL & MQ & $\mathrm{F}_{\text {calculado }}$ & $\mathrm{F}_{\text {tabelado }}(\mathrm{p} \leq 0,05)$ & $\mathrm{R}^{2}$ \\
\hline Regressão & 10,2496 & 3 & 3,4165 & 48,05 & 4,35 & 0,9537 \\
\hline Resíduos & 0,4976 & 7 & 0,0711 & & & \\
\hline F. ajuste & 0,4263 & 5 & 0,0853 & 2,39 & 19,3 & \\
\hline E. puro & 0,0713 & 2 & 0,0357 & & & \\
\hline Total & 10,7472 & 10 & & & & \\
\hline
\end{tabular}

SQ: soma quadrática; GL: graus de liberdade; e MQ: média quadrática. 


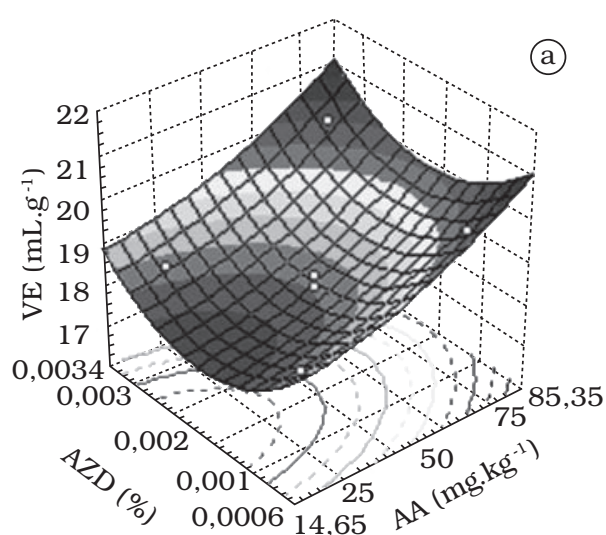

(b)

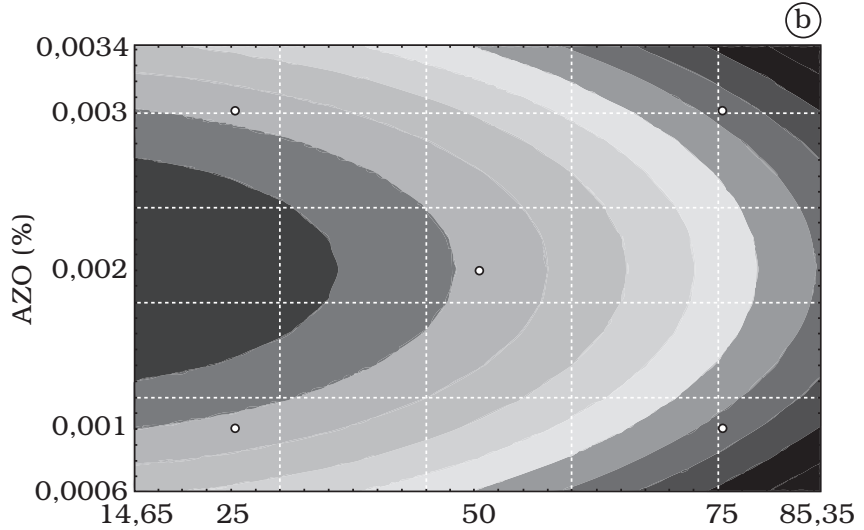

Figura 1. a) Superfície de resposta quadrática; e b) curvas de nível para o volume específico do pão francês.

da resposta para cada parâmetro estudado (ácido ascórbico e azodicarbonamida). Observa-se que a região onde são obtidos os maiores valores de volume específico corresponde às concentrações de ácido ascórbico superiores a $75 \mathrm{mg} . \mathrm{kg}^{-1}$, mesmo para baixas concentrações de azodicarbonamida. Como já foi observado na análise de efeitos, somente verifica-se a influência da azodicarbonamida em concentrações maiores que $30 \mathrm{mg} \cdot \mathrm{kg}^{-1}$, evidenciando o efeito quadrático desse fator no volume específico do pão francês.

De acordo com a análise das curvas de nível, não houve efeito de interação entre o ácido ascórbico e a azodicarbonamida dentro dos níveis estudados no planejamento experimental. Economicamente, verifica-se a vantagem da substituição total do ácido ascórbico por azodicarbonamida, desde que usado em concentrações superiores a $30 \mathrm{mg} \cdot \mathrm{kg}^{-1}$, em relação a farinha de trigo, e sem ultrapassar o limite máximo (40 mg. $\mathrm{kg}^{-1}$ ) estabelecido pela legislação vigente ${ }^{4}$.

\section{Pontuação total das características} externas $e$ internas do pão francês

Os resultados da análise estatística, aplicados aos dados da pontuação total das características externas e internas do pão francês, não apresentaram efeitos significativos em nível de $95 \%$ de confiança ( $\mathrm{p} \leq 0,05$ ) ou valores próximos. Isso mostra que essa variável dependente, dentro das condições estudadas, não representa uma resposta importante à avaliação da influência do ácido ascórbico e da azodicarbonamida na qualidade do pão francês.

\section{Conclusões}

Os fatores que apresentaram efeitos significativos $(\mathrm{p} \leq 0,05$ ou valores próximos) para o aumento do volume específico do pão francês foram: ácido ascórbico (linear e quadrático) e azodicarbonamida (quadrático).

Dentro dos níveis estudados, a aplicação de teores de ácido ascórbico e azodicarbonamida acima de 75 e 30 mg. $\mathrm{kg}^{-1}$, respectivamente, proporcionaram o maior aumento do volume específico do pão francês.

\section{Referências bibliográficas}

1. AACC. American Association of Cereal Chemists. Approved Methods, 10. ed. St. Paul: AACC, 2000.

2. ATWELL, W. A. Wheat flour: practical guide for the food industry. St. Paul: American Association of Cereal Chemists, 2001, 134p.

3. BARROS NETO, B.; SCARMINIO, I. S.; BRUNS, R. E. Planejamento e Otimização de Experimentos, 2. ed., Campinas: Editora da Universidade Estadual de Campinas, 1996, 299p.

4. BRASIL. Resolução no 383, de 05 de agosto de 1999. Regulamento técnico que aprova o uso de aditivos alimentares, estabelecendo sua funções e seus limites máximos para a categoria de alimentos 7 - produtos de panificação e biscoitos. Diário Oficial da República Federativa do Brasil, Brasília, DF, 09 ago. 1999. Disponível em: http://e-legis.anvisa.gov.br/leisref/public/showAct. php?id=117. Acesso em: 06 jun. 2006.

5. BRASIL. Resolução RDC no 263, de 22 de setembro de 2005 . Regulamento técnico para produtos de cereais, amidos, farinhas e farelos. Diário Oficial da República Federativa do Brasil, Brasília, DF, 23 set. 2005. Disponível em: http://e-legis.anvisa. gov.br/leisref/public/showAct.php?id=18822\&word=. Acesso em: 06 jun. 2006.

6. DANNO, G.; HOSENEY, R. C. Effect of sodium chloride and sodium dodecyl sulfate on mixograph properties. Cereal Chemistry, v. 59, n. 3, p. 202-204, 1982.

7. DUBOIS, D. K. Fermented doughs. Cereal Foods World, v. 26, n. 11, p. 617-622, 1981.

8. EL-DASH, A. A. Standardized mixing and fermentation procedure for experimental baking test. Cereal Chemistry, v. 55, n. 4 , p. 436-446, 1978.

9. EL-HADY, E. A. et al. Changes in gas production and retention in non-prefermented frozen wheat doughs. Cereal Chemistry, v. 73 , n. 4, p. 472-477, 1996.

10. FISCHER, F. Oxidation and reduction electron-transfer is key to dough improvements. Bakers Digest, Beloit: Siebel Institute of Technology, v. 59, n. 1, p. 22, 1985.

11. FITCHETT, C. S.; FRAZIER, P. J. Action of oxidants and other improvers. In: Chemistry and Physics of Baking. BLANSHARD, J. M. V.; FRAZIER, P. J.; GALLIARD, T. (ed.). The Royal Society of Chemistry, London. p. 179-198, 1987.

12. LANG, C. E.; NEISES, E. K. ; WALKER, C. E. Effects of additives on flour-water dough mixograms. Cereal Chemistry, v. 69, n. 6, p. 587-591, 1992. 
13. SALAS-MELLADO, M. M. Efeito de algumas variáveis do processo de panificação de massa congelada na estabilidade da massa estocada e na qualidade do pão. 1992. Dissertação (Mestre em Tecnologia de Alimentos), Faculdade de Engenharia de Alimentos, Universidade Estadual de Campinas, UNICAMP, Campinas.

14. STATISTICA for Windows - Release 5.0 A. StatSoft, Inc. Tulsa, OK, USA, 1995.

15. STAUFFER, C. E. Dough conditioners. Cereal Foods World, v. 28, n. 12, p. 729-730, 1983.
16. STAUFFER, C. E. Functional additives for bakery products. New York: AVI, 1990. p. 1-40.

17. STEAR, C. A. Handbook of Breadmaking Technology. London: Elsevier Applied Science, 1990.

18. YAMADA, Y.; PRESTON, K. R. Sponge-and-dough bread: effects of oxidants on bread and oven rise properties of a canadian red spring wheat patent flour. Cereal Chemistry, v. 71, n. 3 , p. 297-300, 1994.

19. WOOD, P. Compound dough conditioners. In: BROWN, J. (ed.), The Master Bakers: Book of Breadmaking, 2. ed. Hertfordshire: Hastings Printing Co., p. 78-108, 1985. 